\title{
Surface Plasmon States in Inhomogeneous Media at Critical and Subcritical Metal Concentrations
}

\author{
Katyayani Seal ${ }^{1}$ and Dentcho A. Genov ${ }^{2}$ \\ ${ }^{1}$ Department of Physics and Astronomy, University of Tennessee, Knoxville, TN 37996, USA \\ ${ }^{2}$ College of Engineering and Science, Louisiana Tech University, Ruston, LA 71270, USA
}

Correspondence should be addressed to Katyayani Seal, sealk@ornl.gov

Received 30 September 2011; Accepted 14 December 2011

Academic Editor: Ali Passian

Copyright @ 92012 K. Seal and D. A. Genov. This is an open access article distributed under the Creative Commons Attribution License, which permits unrestricted use, distribution, and reproduction in any medium, provided the original work is properly cited.

Semicontinuous metal-dielectric films are composed of a wide range of metal clusters of various geometries-sizes as well as structures. This ensures that at any given wavelength of incident radiation, clusters exist in the film that will respond resonantly, akin to resonating nanoantennas, resulting in the broad optical response (absorption) that is a characteristic of semicontinuous films. The physics of the surface plasmon states that are supported by such systems is complex and can involve both localized and propagating plasmons. This chapter describes near-field experimental and numerical studies of the surface plasmon states in semicontinuous films at critical and subcritical metal concentrations and evaluates the local field intensity statistics to discuss the interplay between various eigenmodes.

\section{Introduction}

Random metal-dielectric composites, such as nanoscaled semicontinuous metallic structures, exhibit unique optical and electrical transport properties [1-26]. One of the most remarkable properties of such composites is the enhancement of electric and magnetic fields in the visible and infrared spectral ranges $[1,8-22]$. These materials show promise in wide ranging photonics technologies including applications in negative index materials [27-29], biosensing [30, $31]$, and nonlinear optics [10] spectroscopy [32, 33] broadband optical amplifiers and optical absorbers $[10,34,35]$.

The synthesis of metal-dielectric composites typically involves the deposition of metal particles onto a dielectric substrate [14, 21-23]. At low metal concentrations, mutually separated nanometer-sized grains are formed at random locations. Self-similar clusters form as the metal surface concentration increases $[1,3,10]$. At a certain metal concentration $\left(p_{c}\right)$, that is, the percolation threshold, the metal clusters interconnect to form an infinite "backbone cluster", setting off an insulator-to-metal phase transition. This is marked by a sharp drop in dc resistivity $[14,23]$ and anomalous absorption at visible and near-infrared wavelengths $[1,10,11,14-19,21-23]$. At even higher metal concentrations, the sample becomes mostly metallic with dielectric voids, ultimately resulting in a uniform metal film.

From both theoretical predictions and experimental corroboration, it has been observed that under illumination with light at optical and near infrared frequencies, strong local field enhancement of up to $10^{4}$ can be achieved on the surface of two-dimensional metal-dielectric composites [1, 10-22, 36]. This light trapping effect is facilitated by the excitation of collective electron oscillations, that is, surface plasmons (SP). Surface plasmon modes are morphological resonances governed by the structural inhomogeneities inherent in the fractal clusters formed within the random metal-dielectric films. The observed local fields appear to be strongly localized $[1,10,11,17-19]$ with relatively high Q-factors dependent on the intrinsic dissipation in the metal. This phenomenon has been observed in rough and semicontinuous metal films $[8,9,15,16,23]$. Notably, due to the geometry of the clusters, the spatial distribution of the field maxima is sensitive to the polarization, wavelength and angle of incidence of the applied field $[1,2,10]$. Thus the 
metal clusters in a semicontinuous film that support SP modes may be thought of as resonating nanoantennas with a range of resonant frequencies and Q-factors. The accumulation and subsequent dissipation of electromagnetic energy in these localized SP modes result in the anomalous absorption characteristic of semicontinuous metal films $[1,2,5-$ $10,14-22]$.

The near-field intensity distribution across a semicontinuous metal film near the "percolation threshold" has been observed to be extremely inhomogeneous with giant local field maxima $[15,16,20-22]$, that vary not only in size by orders of magnitude from subwavelength to multiple wavelengths, but also vary strongly in intensity. In the early theoretical studies on percolating metal-dielectric composites, an observed similarity between the Kirchhoff Hamiltonian used to model random metal dielectric systems and the Anderson Hamiltonian [37] led to the expectation that all surface plasmon eigenmodes are localized [10]. Follow-up numerical simulations subsequently suggested that a planar disordered nanosystem can simultaneously have localized and delocalized states [18]. This concept of "inhomogeneous localization" was originally introduced for metal fractal aggregates, where self-similarity in the structural geometry can result in a mode consisting of multiple hot spots spread over the entire system [10]. Later calculations have shown that in an infinitely large semicontinuous metal film, delocalized SP modes exist only at one point in the eigenvalue spectrum and their measure is zero [19]. The conceptual origin for these delocalized states lies in the short-range correlations in the governing Kirchhoff Hamiltonian, which are absent in the Anderson Hamiltonian [37]. Remarkably, even though they have a zero measure, delocalized modes can affect the local field statistics by modifying the critical indices for the high-order field moments $[19,22]$ and thus influence the optical properties. The interplay between localized and delocalized states in semicontinuous films and their role in the transport properties therein remain a topic of interest and speculation.

For disordered systems with a range of possible eigenmodes and transport paths, studies of field and intensity statistics are a valuable avenue to uncover the system's dominant response and the nature of wave transport. In disordered metallic nanostructures in particular, the intensity statistics are more complex because of the excitation of surface plasmons and resulting inhomogeneous localization [18]. In the study of random metal-dielectric composites, both numerically $[2,14,17-19]$ and experimentally $[5-7,15,16,19-$ 23] obtained near-field intensity statistics are often used to characterize the local field fluctuations in various metal aggregates, fractal structures, and rough and semicontinuous metal films.

This chapter provides a comprehensive view of the interplay between localized and delocalized plasmon excitations in metal semicontinuous films through the analysis of experimentally and numerically obtained near-field intensity statistics.

\section{Synthesis of Semicontinuous Films and Near-Field Statistics}

Semicontinuous silver films on glass substrates are typically synthesized by thermal evaporation [5-9] or by pulsed laser deposition [16]. Transmission electron microscope (TEM) images from a silver-glass semicontinuous film grown by pulsed laser deposition are shown in Figure 1(a). From the images, it is evident that these samples are composed of individual silver grains of average size about $20-30 \mathrm{~nm}$. An increase in deposition time (surface concentration of silver) induces a structural transition from isolated metal grains $(p<0.4)$ to interconnected metal clusters $(p=0.6)$ and finally to a nearly continuous metal film with dielectric voids $(p>0.8)$ [16]. For these samples, the percolation threshold was found to be at $p_{c}=0.65$ from previous work [16]. The $2 \mathrm{D}$ structural correlation functions were found to be isotropic. The correlation radius, defined as the half width at half maximum (HWHM) of the structural correlation function, is close to the grain size $\sim 20 \mathrm{~nm}$.

Near-field optical microscopy (NSOM) is a powerful tool to image the near-field intensity distribution over the surface of a sample $[15,16]$. Figure 1 (b) shows NSOM images for silver on glass semicontinuous films with metal concentration $p=0.36,0.45,0.65,0.75$, and 0.83 at a probe wavelength of $543 \mathrm{~nm}$. The samples were illuminated by the evanescent field (in the total internal reflection geometry) of "p" polarized laser light. The projection of the incident beam's $\mathbf{k}$ vector on the film plane ( $x-y$ plane), $\mathbf{k}_{\|}$, is along the vertical $y$ axis. The local optical signal was collected by a tapered, uncoated optical fiber of tip radius of about $50 \mathrm{~nm}$. The tipto-sample distance, controlled by shear-force feedback, was approximately $10 \mathrm{~nm}$. The tip resolution is estimated to be of the order of $150 \mathrm{~nm}$ from the smallest features in the nearfield images $[16,22]$. The images exhibit inhomogeneous intensity distributions with intensity maxima (or optical "hot spots") of various sizes. At $p=0.36$, there is an indication of interference fringes parallel to the horizontal $x$ axis, which are weakened at $p=0.45$, completely lost at $p=0.65$, and reappear at $p=0.83$.

To understand the role of the eigenmodes supported by various clusters in the semicontinuous film, it is instructive to study the near-field intensity statistics for different metal concentrations $p$. Specifically, the variance $\operatorname{var}(I)=$ $\left\langle I(x, y)^{2}\right\rangle /\langle I(x, y)\rangle^{2}-1$ and higher-order moments $M_{2 n}=$ $\left\langle I(x, y)^{n}\right\rangle / I_{0}{ }^{n}$ can be obtained from the NSOM images, where $I(x, y)$ is the intensity over the sample surface as a function of $x$ and $y$ coordinates. Figure 2 shows the experimentally obtained values of $\operatorname{var}(I)$ as a function of $\Delta p=p-p_{c}$ at a probe wavelength of $633 \mathrm{~nm}$ calculated from the NSOM images similar to the ones in Figure 1(b). Each data point was obtained from approximately 300,000 near-field intensity values. The error bar was evaluated as the standard error of data collected from several NSOM images of different parts of the samples. Counterintuitively, the intensity fluctuations do not maximize at $p_{c}(\Delta p=0)$, where scattering is the strongest. Instead, a local minimum appears at $p_{c}$. Away from $p_{c}, \operatorname{var}(I)$ has two maxima at $\Delta p=-0.2$ and $\Delta p=+0.1$. A similar dependence of $\operatorname{var}(I)$ 


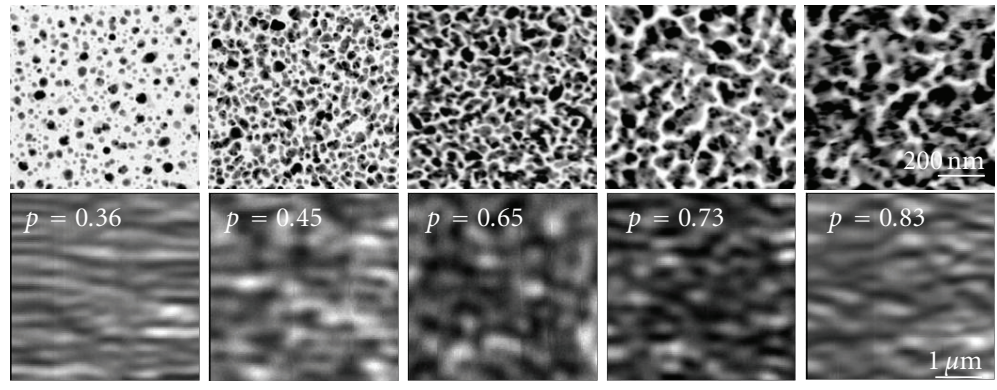

Figure 1: TEM micrographs of silver semicontinuous films on glass substrates showing the metal nanostructure geometry for various metal concentrations in the first row. The second row shows the inhomogeneous distribution of the near-field intensity for corresponding samples.

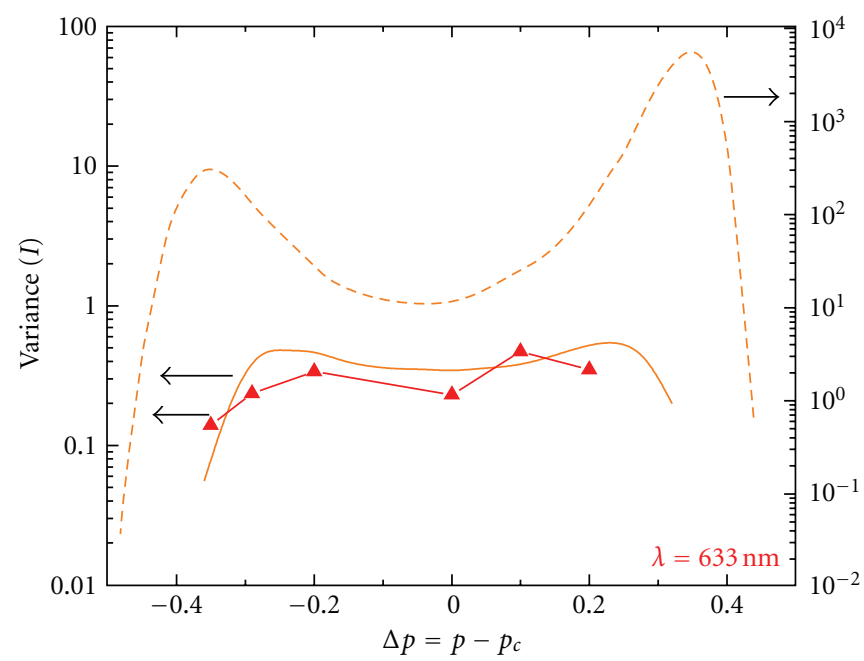

FIGURE 2: Near-field intensity variance $\operatorname{var}(I)$ as a function of silver concentration $\Delta p=p-p_{c}$, obtained experimentally (solid line with triangles) and numerically with (dotted line) and without (dashed line) spatial averaging [22, Figure 1].

on $p$ is also observed at lower probe wavelengths $(543 \mathrm{~nm})$. However, the absolute value of $\operatorname{var}(I)$ at $\lambda=543 \mathrm{~nm}$ is lower than that at $\lambda=633 \mathrm{~nm}$, indicating that the intensity fluctuations are stronger at longer wavelengths.

The variance of intensity fluctuations is proportional to the inverse participation ratio (IPR) of the local field, often used to describe the spatial extent of eigenstates [2]. In the experimental set up described, where the incident field excites a number of SP modes, IPR gives a qualitative measure of the surface area occupied by hot spots. Similar to $\operatorname{var}(I)$, IPR has a minimum at $p_{c}$. Larger values of IPR at $\left|p-p_{c}\right|=0.1$ and 0.2 indicate that a smaller percentage of the film surface is occupied by hot spots. This is consistent with the direct observation that away from $p_{c}$, there are fewer hot spots but each is brighter.

The experimentally observed dip in IPR suggests a weakening of the SP localization strength at $p_{c}$. For the calculation of the high-order moments of intensity enhancement $M_{2 n}$, the incident light intensity $I_{0}$ obtained from the near-field measurement of a clean glass substrate can be used as a standard normalizing factor. Figure 3(a) is a plot of $M_{2 n}$ versus $\Delta p$ for $n=2,3,4$, and 5 obtained from the same NSOM images (at a probe wavelength of $633 \mathrm{~nm}$ ) as were used in Figure 2. The error bars were obtained by the same method as for $\operatorname{var}(I)$ in Figure 2. The $M_{2 n}$ exhibit a prominent dip near $p_{c}$, which becomes more pronounced for higher $n$. This observation once more confirms that the local field fluctuations are weakened at the percolation threshold.

\section{Numerical Model of a Semicontinuous Film}

In order to understand the experimental findings, it is useful to perform numerical simulations to simulate the semicontinuous film. In the visible frequency regime, metal-dielectric composites can be viewed as a system of lumped $R L C$ circuits $[2,10]$, and the bond percolation model can be employed to describe their electromagnetic response. The bond model has a percolation threshold $p_{c}=0.5$, which differs slightly from the experimental observation. This deviation, however, should not preclude a direct mapping between experiment and theory because the scaling properties of random metal films do not depend on the absolute value of $p_{c}$ [3].

The inhomogeneous metal dielectric composite is a random system of metal nanoparticles incorporated within a dielectric host and can be modeled as a strongly coupled lumped $R-L-C$ network. Provided the feature sizes along 


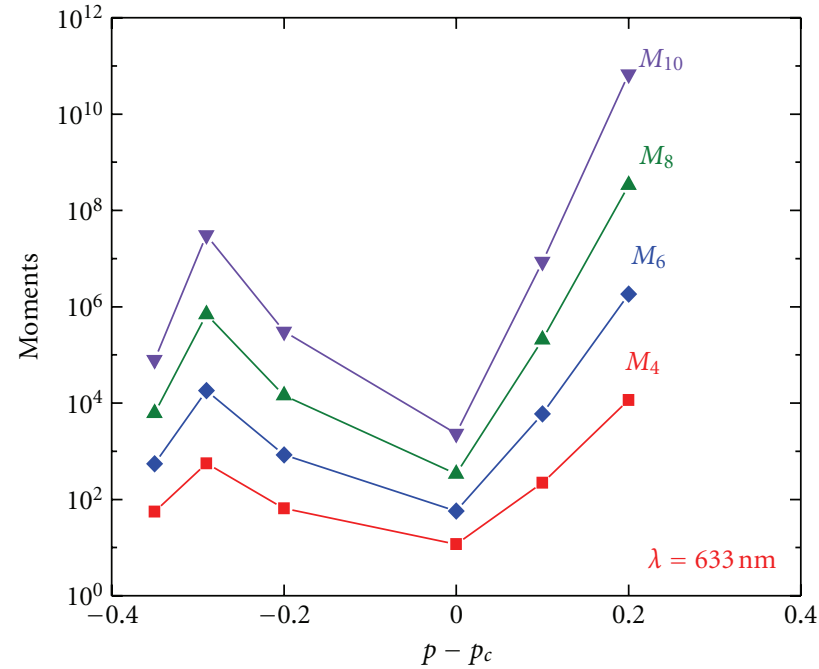

(a)

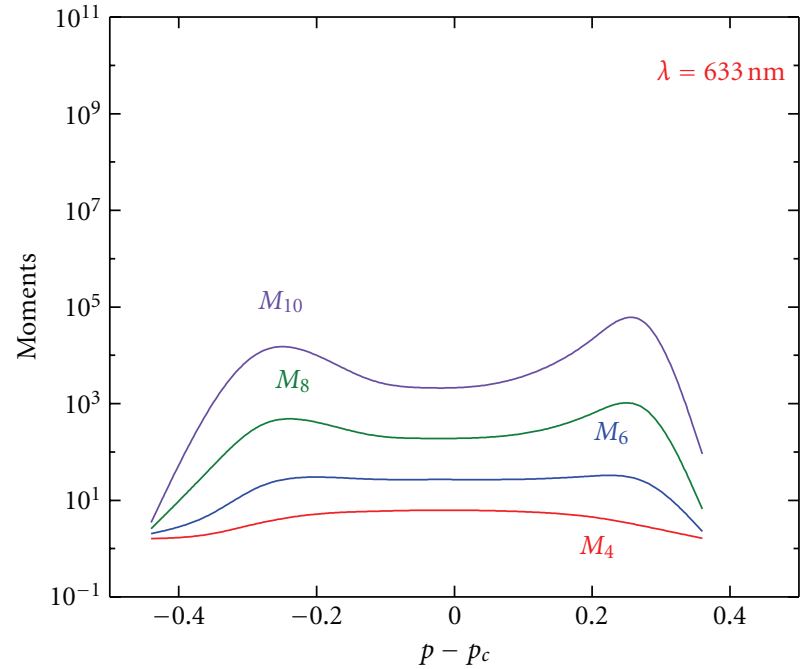

(b)

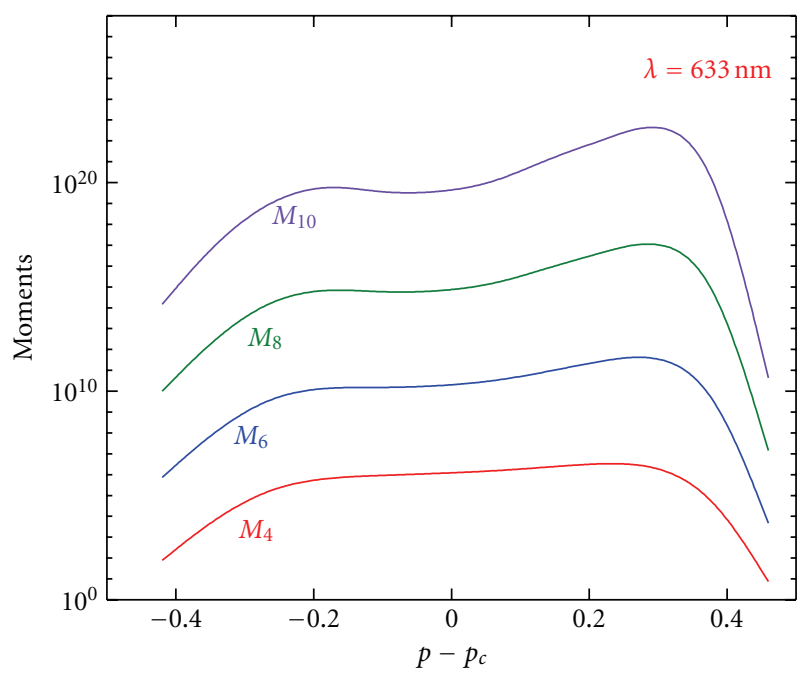

(c)

Figure 3: High-order moments of near-field intensity enhancement $M_{2 n}$ as a function of silver concentration $\Delta p=p-p_{c}$, obtained experimentally (a) and numerically with (b) and without (c) spatial averaging [22, Figure 2].

the direction of illumination are small compared to the incident wavelength $\lambda=2 \pi c / \omega$, the impinging electric field $\vec{E}_{0}$ couples with the metal dielectric composite quasistatically, with a local electric potential $\varphi(\omega)$ given as

$$
\vec{\nabla} \cdot[\sigma(\omega, \vec{r}) \vec{\nabla} \varphi(\vec{r})]=\vec{E}_{0} \cdot \vec{\nabla} \sigma(\omega, \vec{r}),
$$

where the local conductivity $\sigma(\omega, \vec{r})$ is a discontinuous random function across the composite taking discrete values $\sigma_{m}=-i \omega \varepsilon_{m}$ for the metal particles and $\sigma_{d}=-i \omega \varepsilon_{d}$ for the dielectric voids with probabilities $p$ and $1-p$, respectively [14]. Equation (1) describes a generalized Kirchhoff problem and can be solved numerically by discretization of the electric potential on a cubic lattice, with the metal and dielectric constituents represented by randomly distributed metal/dielectric bonds connecting each lattice site. The resulting linear system of equations is written in short form
$\hat{H} \cdot \vec{\varphi}=\widehat{W} \cdot \hat{E}_{0}$, where $\hat{H}$ is a complex value Kirchhoff Hamiltonian $(\mathrm{KH}), \vec{\varphi}=\left\{\varphi_{i, j}\right\}$ is a vector containing the values of the potential at each lattice side $(i, j)$, and $\widehat{W}$ is the incident field projection operator. The Kirchhoff Hamiltonian is a random matrix with diagonal elements $H_{n n}=$ $\sum \sigma_{\{i, j\}_{n}}$, where the summation is over all bond conductivities connecting the $n$th site with its nearest neighbors, and correlated off-diagonal elements proportional to the respective bond conductivities $\sigma_{\{i, j\}_{n}}$. Traditional numerical methods such as Gaussian eliminations require $O\left(L^{6}\right)$ floating point operations and $O\left(L^{4}\right)$ operational memory to achieve solution (here $L$ is the lateral size of the composite). For the system sizes of interest (a few $\mu m^{2}$ or up to a million metal particles), these algorithms are not feasible even with high performance computing. To overcome this computational problem, a block elimination method has been developed that splits the Kirchhoff Hamiltonian on $L \times L$ block matrixes 
and performs triangulation on these separated block of matrixes [2]. This effectively allows the reduction of this twodimensional problem to an equivalent 4/3-dimensional problem, resulting in dramatic improvement in performance with solution obtained in only $O\left(L^{4}\right)$ floating point operations and operational memory requirement of the order of $O\left(L^{3}\right)$.

The numerical results based on the Block Elimination procedure are shown in Figures 2, 3(b), and 3(c), where the local field distribution is calculated for $2 \mu m \times 2 \mu m$ sized semicontinuous films of different silver concentrations, followed by calculations of $\operatorname{var}(I)$ and $M_{2 n}$. Detailed information on the method of calculation used is provided in $[2,10]$. At the percolation threshold a highly inhomogeneous local field distribution is observed, with peak intensities surpassing the incident laser light intensity by factors of $10^{3}-$ $10^{4}$.

The experimentally obtained local field enhancements are considerably lower in comparison because of the finite spatial resolution of NSOM. For optimal comparison with the experimental data, the local fields at a distance equal to the tip-sample separation ( $\sim 10 \mathrm{~nm}$ for the NSOM configuration used) from the sample surface can be estimated numerically and then averaged using the NSOM aperture function (in this case, corresponding to a tip resolution of $\sim 150 \mathrm{~nm})$. Numerical data for $\operatorname{var}(I)$ and $M_{2 n}$ before and after the spatial averaging are provided in Figures 2, 3(b), and $3(\mathrm{c})$. These curves were obtained from 100 realizations of the semicontinuous film surface for each $p$. As shown in Figure 2, the $\operatorname{var}(I)$ calculated without spatial averaging exhibits a wider and deeper dip near $p_{c}$. The spatial averaging not only reduces the value of $\operatorname{var}(I)$ but also partially smooths out the dip at $p_{c}$. After the resolution limit of the NSOM is taken into account, the numerical data closely mimic the experimental $\operatorname{var}(I)$. However, some of the numerical values fall outside the experimental error bars because of inevitable approximations used in the calculations such as uncertainty in the value of dielectric constant of silver, the tip-sample distance, and the spatial averaging parameter. In Figure 3, the calculated local field moments $M_{2 n}$ exhibit a $p$-dependence similar to the measured one, that is, $M_{2 n}$ reaches a local minimum at $p_{c}$ and has two distinct maxima at $|\Delta p| \leq 0.3$. The above numerical uncertainties also contribute to the discrepancies between experimental and numerical (spatially averaged) data shown in Figure 2.

\section{A Qualitative Understanding of the Variations in Localization Strength}

The observed decrease of $\operatorname{var}(I)$ and $M_{2 n}$ at $|\Delta p| \geq 0.3$ can be qualitatively explained by considering the resonant nature of SP excitation. According to percolation theory [3], the largest metal cluster in the random composite has a size $l_{m}(p)=a p_{c}^{v} /\left|p-p_{c}\right|^{\nu}$, where $a$ is the average metal particle size and $v$ is a critical exponent. Resonant SP modes can be excited by incident light of a wavelength $\lambda$ if $l_{m}(p) \geq$ $l_{r}=a\left(\left|\varepsilon_{m}^{\prime}\right| / \varepsilon_{d}\right)^{\nu /(s+t)}$, where $\varepsilon_{m}^{\prime}+i \varepsilon_{m}^{\prime \prime}$ and $\varepsilon_{d}$ are the dielectric constants of the metal particles and dielectric host, respectively [10]. The critical exponents $s$ and $t$ correspond to the scaling of the composite's static conductivity and dielectric constant, respectively, with the sample size. For the planar systems under consideration, $s=v=t=4 / 3$ [3]. Thus the SP resonance wavelength in a cluster of size $l \geq l_{r}$ scales as $\lambda_{r} \approx \lambda_{p}(l / a)^{\nu /(s+t)} \approx \lambda_{p} \sqrt{l / a}$, where $\lambda_{p}$ is the plasma wavelength [10]. This unique property of localized SPs is distinct from resonances in the retarded (nonquasistatic) regime where $\lambda_{r} \approx l$.

When the metal concentration $p$ differs considerably from $p_{c}$, there is a decrease in the maximum cluster size $l_{m}$. If $l_{m}(p)<l_{r}$, SP modes in the semicontinuous metal film can no longer be excited by the external field, leading to a dramatic decrease in the local electromagnetic response. The cutoff metal concentrations are estimated from the condition $l_{r}=l_{m}\left(p_{ \pm}\right)$as $p_{ \pm}=p_{c}\left[1 \pm\left(\varepsilon_{d} /\left|\varepsilon_{m}^{\prime}\right|\right)^{1 /(s+t)}\right]$. At $\lambda=633 \mathrm{~nm}$, $\Delta p_{ \pm}=\left|p_{ \pm}-p_{c}\right| \approx 0.3$. For concentrations in the range $|\Delta p|>\Delta p_{ \pm}$, both $\operatorname{var}(I)$ and $M_{2 n}$ are expected to decrease sharply. Estimates for the cutoff concentrations $\Delta p$ agree well with the experimental and numerical results shown in Figures 2 and 3. The presence of cutoff metal concentrations also manifests itself as an anomalous absorption band in semicontinuous metal films [23]. Specifically, when $\kappa=$ $\left|\varepsilon_{m}^{\prime \prime} / \varepsilon_{m}^{\prime}\right| \ll 1$, the buildup of giant local fields and storage of electromagnetic energy in the films leads to strong light absorption, which occurs for metal concentrations inside the band $p_{c}-\Delta p_{-}<p<p_{c}+\Delta p_{+}[26]$. The quantity $\Delta p_{ \pm}$, calculated as $\sqrt{\left|\varepsilon_{m}^{\prime}\right| / \varepsilon_{d}} /\left(\left|\varepsilon_{m}^{\prime}\right|+\varepsilon_{d}\right)$ from effective medium theory, gives values close to the cutoff concentration estimates based on the resonant cluster size.

\section{Density of States and Localization Length from the SP Eigenproblem}

To further understand the behavior of $\operatorname{var}(I)$ and $M_{2 n}$ at $p_{c}$, it is instructive to solve the SP eigenproblem. In order to model the local electric response of a random system comprised of a large number of metal particles, one can work in the quasistatic limit. The general solution of the current conservation (1) can be described completely by examining the underlying eigenproblem $[10,19]$

$$
\vec{\nabla} \cdot\left[\sigma(\vec{r}) \vec{\nabla} \Psi_{\Lambda}(\vec{r})\right]=\Lambda \Psi_{\Lambda}(\vec{r}) .
$$

Reduction of (2) on a square lattice leads to a system of linear equations $\hat{H}_{\mathrm{KH}}|n\rangle=\Lambda_{n}|n\rangle$, where again the Kirchhoff Hamiltonian $\hat{H}_{\mathrm{KH}}=\sum_{n} h_{n n}|n\rangle\left\langle n\left|+\sum_{n \neq k} h_{n k}\right| n\right\rangle\langle k|$ is a random matrix with correlated diagonal and off-diagonal elements and $|n\rangle$ are the SP eigenstates $[5-7,19,16]$. The existence of short range correlations in the SP eigenproblem makes it substantially different than the corresponding quantum mechanical case where the random Hamiltonians are noncorrelated [19]. The correlations in the $\mathrm{KH}$ are due to the local current conservation and exist for all spatial dimensions.

The statistical properties of the solution to the SP eigenproblem can be investigated in terms of the density of states $\rho(\lambda)$ and SP localization lengths $\xi(\lambda)$. These are plotted 


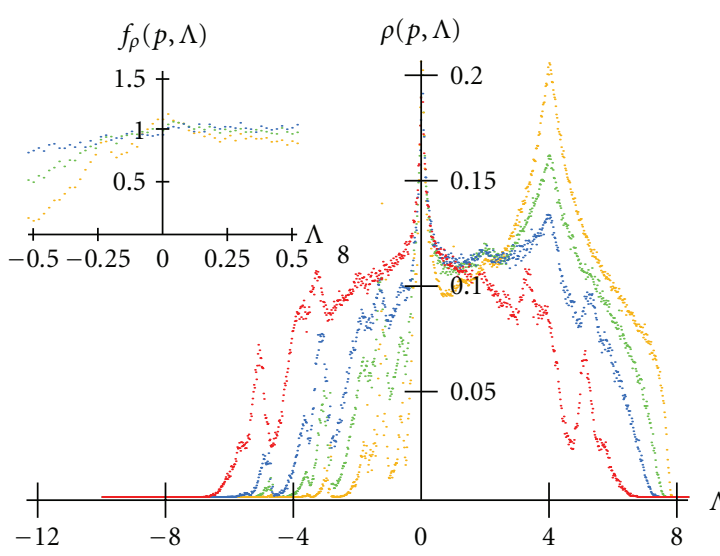

(a)

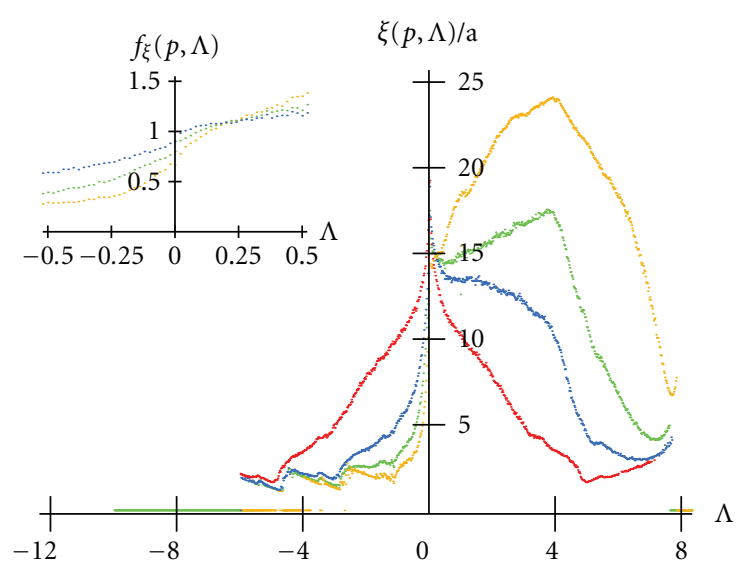

(b)

FIGURE 4: The surface plasmons density of states (DOS) (a) and localization length (b) are calculated for an ensemble of 100 metal-dielectric films each with size $1 \mu \mathrm{m}$. Four different metal filling fractions are investigated: $p=0.1$ (yellow color), $p=0.2$ (green color), $p=0.3$ (blue color), and $p=0.5$ (red color). The singularity at the band center and its dependence on the metal concentration is closely inspected in the insets [24, Figure 2].

for different metal concentrations in Figures 4(a) and 4(b), respectively. At the band center, and concentrations close to percolation, the density of states exhibits a singularity as shown in Figure 4(a). In the first approximation the density of states seems to diverge as a power law $\rho(\Lambda) \sim|\Lambda|^{-\gamma}$ at $p=p_{c}$ (red curve), where $\gamma=0.14 \pm 0.01$ [17] is a critical exponent. The localization length for each eigenmode (presented in Figure 4(b)) is also calculated using the gyration radius $\xi^{2}(p, \Lambda)=\int\left(\vec{r}-<\vec{r}>_{\Lambda}\right)^{2}\left|\Psi_{\Lambda}(\vec{r}, p)\right|^{2} d \vec{r}$, where $<\vec{r}>_{\Lambda}=\int \vec{r}\left|\Psi_{\Lambda}(\vec{r}, p)\right|^{2} d \vec{r}$ is the "mass center" of the $n$th mode and the integration is performed over the film surface. Similar to the density of states, there is a singularity at the band center. At $p_{c}$, the localization length diverges logarithmically for $\Lambda \rightarrow 0$, but also can be fitted with a power law $\xi(\Lambda) \sim|\Lambda|^{-\alpha}$, where $\alpha=0.15 \pm 0.02$.

Relying on the SP eigenproblem (2), it is possible to estimate the optical response of the random metal films for an incident frequency $\omega_{r}$ corresponding to the single particle resonance Re $\varepsilon_{m}\left(\omega_{r}\right)=-\varepsilon_{d}$. Specifically, the local potential is expanded over the SP eigenspace [10] to obtain an integral relationship for the local field moments

$$
\begin{aligned}
M_{2 n} & =\frac{1}{S\left|E_{0}\right|^{2 n}}\left\langle\int|E(\vec{r}, p)|^{2 n} d \vec{r}\right\rangle \\
& \cong \int_{-\infty}^{\infty} \frac{\rho(p, \Lambda)[a / \xi(p, \Lambda)]^{2(2 n-1)}}{\left(\Lambda^{2}+\kappa^{2}\right)^{n}} d \Lambda,
\end{aligned}
$$

where $\kappa=\left|\varepsilon_{m}^{\prime \prime} / \varepsilon_{m}^{\prime}\right| \ll 1$ is the intrinsic loss factor, $S$ is the sample area, and the brackets correspond to ensemble averaging over large numbers of system realizations.

The integral in (3) can be analyzed as follows. Close to the band center $(|\Lambda| \ll 1)$, the properties of the generalized SP eigenstates can be modeled (see inserts in Figure 4 ) by simplified expressions for the SP DOS $f_{p}(\rho, \lambda)=\rho(p, \lambda) /$ $\rho\left(p_{c}, \lambda\right) \approx 1$ and the SP localization length $f_{\xi}(p, \Lambda)=\xi(p$, $\Lambda) / \xi\left(p_{c}, \Lambda\right) \approx g(p)+\theta(\Lambda)(1-g(p))$, where $\theta(\Lambda)$ is a step function and $g(p)<1$. Since only the SP eigenmodes in the vicinity of the band center have a dominant contribution to the moments, the $p$ dependence from the integral (3) can then be taken out to give $M_{2 n}(p) \approx M_{2 n}\left(p_{c}\right)[1+$ $\left.g^{4(1+n)}(p)\right]$, where $M_{2 n}\left(p_{c}\right)$ are the local field moments at percolation [19]. Using $g(p)<1$, the limiting condition $M_{2 n}(p)>M_{2 n}\left(p_{c}\right) g^{4(1-n)}(p)>M_{2 n}\left(p_{c}\right)$ is obtained, which implies that at $p_{c}$ and for $n>1$, the local field moments have a minimum. This simple reasoning based on the general properties of the SP eigenstates provides a qualitative explanation of the observed dip in the field moments as per Figure 3.

From Figure 4(a), it is also clear that for $p<p_{c}$ the localization lengths of the excited SP eigenstates, predominantly those with $\Lambda<0$, is lower as compared to the critical case $\left(p=p_{c}\right)$. This suggests that at noncritical metal concentrations the role of the localized states in the local field statistics becomes stronger. Moreover, since current conservation holds in all cases, one may expect maxima in $\rho(\lambda)$ and $\xi(\lambda)$ at noncritical metal concentrations as well, as shown in Figures 4(a) and 4(b). With the decrease of $p$, a shift in the DOS is observed with an increase of the number of eigenstates with positive eigenvalues. This phenomenon corresponds to the fact that the eigenproblem maps the underlying film geometry with positive eigenvalues corresponding to eigenstates situated mostly in the dielectric host. It should be pointed out that although Figure 4 presents only the results for $p<p_{c}$, the properties of the SP eigenstates at high and low metal concentrations are equivalent due to symmetry. Thus, the relationships $\rho\left(\Lambda, p_{c}+\Delta p\right)=\rho\left(\Lambda, p_{c}-\Delta p\right)$ and $\xi\left(\Lambda, p_{c}+\Delta p\right)=\xi\left(\Lambda, p_{c}+\Delta p\right)$ hold for any given $\Delta p \leq p_{c}$.

\section{Behavior of the Slope of the Higher-Order Moments $\left(M_{2 n}\right)$}

The increased contribution of the localized states at $p \neq p_{c}$, or alternatively the increase of SP delocalization at $p_{c}$, is revealed through the dependence of $\operatorname{In}\left[M_{2 n}(p)\right]$ on the order 


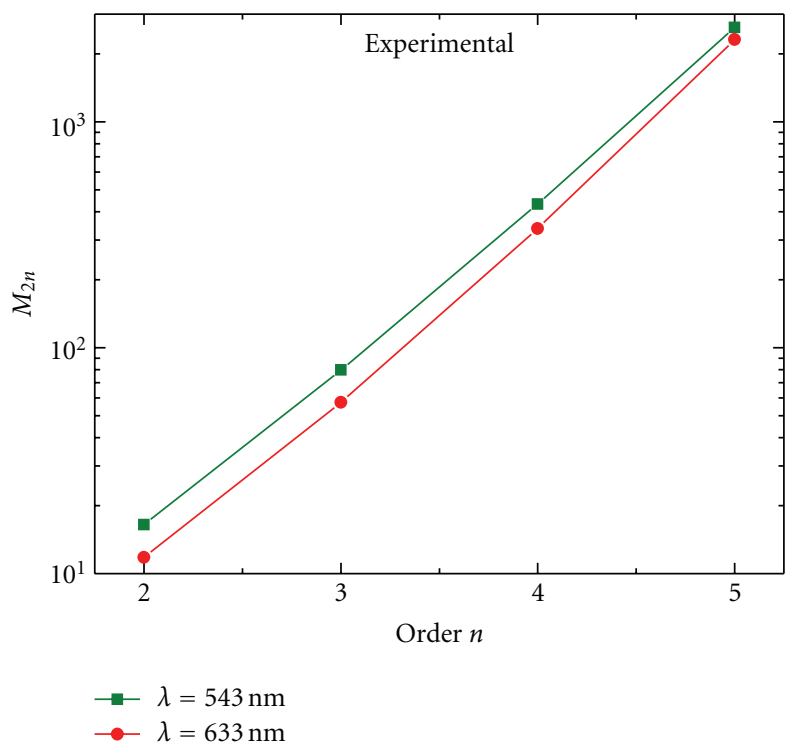

(a)

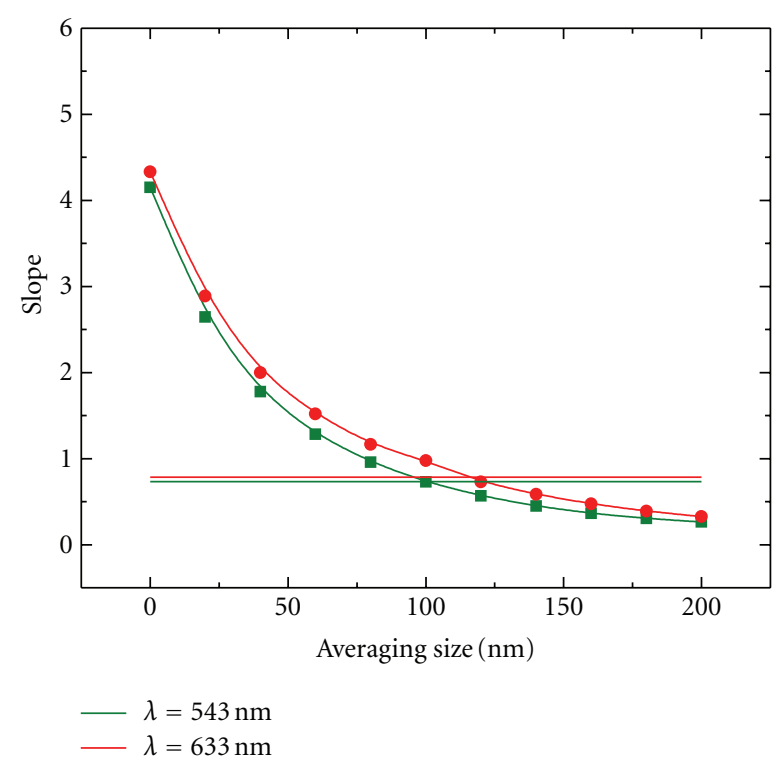

(b)

FIGURE 5: (a) Log-linear plot of measured high-order moments of near-field intensity enhancement $M_{2 n}$ versus $n$. (b) Slope of log( $\left.M_{2 n}\right)$ over $n$ at the percolation threshold obtained numerically after spatial averaging versus averaging parameter $d$. The measured slopes are marked by horizontal lines with the standard error given in the legend [22, Figure 3].

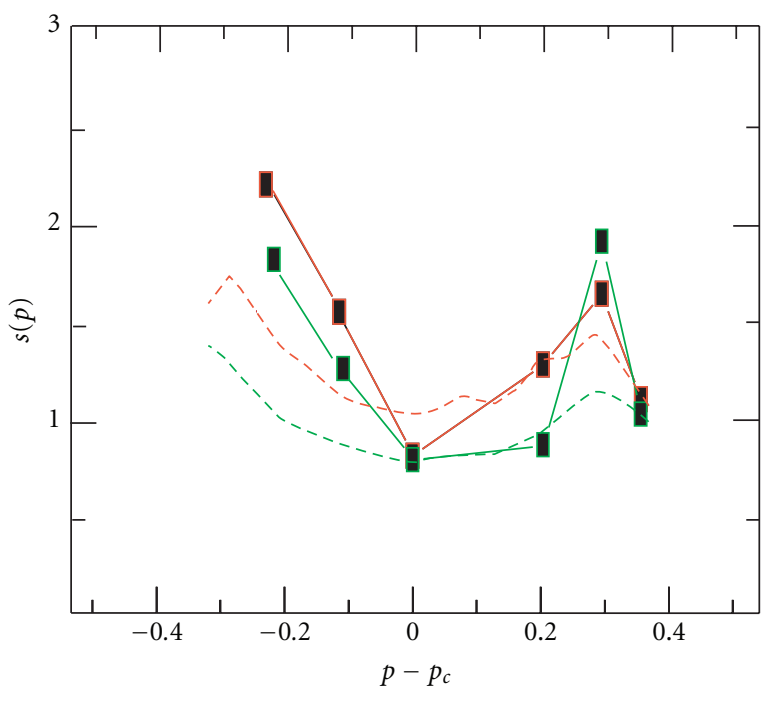

FIGURE 6: Slope versus metal concentration for numerical (dashed line) and experimental (solid line and square symbols) data. The wavelengths of the incident light are $\lambda=543 \mathrm{~nm}$ (green color) and $\lambda=633 \mathrm{~nm}$ (red color) [24, Figure 3].

$n$. According to the scaling theory described above, a linear dependence of the form $\left.\operatorname{In}\left[M_{2 n}(p)\right] \sim 2 n s(p)\right]$ is to be expected, with the slope $s(p)=s\left(p_{c}\right)+4 \operatorname{In}[1 / g(p)] \geq s\left(p_{c}\right)$ increasing for metal concentrations away from the percolation threshold. Figure 5(a) is a log-linear plot of the measured $M_{2 n}$ versus order $n$ at $p_{c}$ for two probe wavelengths $543 \mathrm{~nm}$ and $633 \mathrm{~nm}$. Clearly, a linear dependence on the field moment $n$ is observed. However, due to the limited NSOM resolution, the slopes obtained experimentally were found to be considerably lower than those obtained numerically. To account for the effect of the finite spatial resolution in the experiment, the calculated local field distribution was first averaged over an area of dimension $d$, and then $M_{2 n}$ was computed to extract the slope of $\operatorname{In}\left[M_{2 n}(p)\right]$ versus $n$. Figure 5(b) shows that the slope decreases as $d$ increases. When the slope is reduced to the experimental values (marked by horizontal lines), the averaging parameter $d$ is found to be very close to the NSOM resolution limit estimated from the smallest features in the near-field images. The same value of $d$ also leads to good agreement between the calculated $\operatorname{var}(I)$ and the experimental data.

To extend this result for metal concentrations away from $p_{c}$, Figure 6 plots the slope as described above for various metal concentrations. In the calculations, the local field is again spatially averaged to account for the finite NSOM resolution. Figure 6 shows a similar trend with respect to $p$ for both experiment and numerical calculations, with a clear minimum at $p_{c}$. Although the exact numerical values of the slope at $p \neq p_{c}$ cannot be easily interpreted based only on the SP eigenproblem, the observed increase in slope away from $p_{c}$ clearly indicates a transition at $p_{c}$. The analysis following (3) suggests that stronger SP localization is favored at these metal concentrations, which leads to stronger local field fluctuations. The higher-order moments are more sensitive to those fluctuations, a fact that is manifested by the increase in the slope. It must be pointed out that for $p>p_{c}$, apart from the quasistatic SP modes caused by the sub-wavelength features in the system, the medium could support propagating surface waves and surface plasmon polaritons [20]. However, the local fields modulations that are associated with those propagating modes are weaker 
compared to the quasistatic resonances and should have a relatively small effect on the local field distributions. This conclusion is indirectly supported by the good correlation between the numerical calculations and experiment.

\section{Conclusion}

The implications of the above statistical analysis in practical applications are wide-ranging. The high-order local field moments $M_{2 n}$ correspond to the enhancement of multiphoton optical processes due to the excitation of SP resonances in the substrate. For instance, $M_{4}$ relates to the electromagnetic enhancement of Raman-scattering provided that Raman active molecules are deposited on the metal surface. Since $M_{2 n}(p) \geq M_{2 n}\left(p_{c}\right)$, one may conclude that contrary to common practice, a highly effective surface enhanced Raman substrate can be achieved at low and high metal concentration. A similar conclusion can be made for the higherorder optical processes such as hyper-Raman scattering, Kerr optical nonlinearity, and $n$th harmonic generation.

To conclude, the near-field intensity statistics in semicontinuous silver films over a range of metal concentrations is effective in providing an overview of the various eigenstates supported by the film and the interplay between localized and delocalized SP eigenmodes. The variance of intensity fluctuations and the high-order moments of intensity enhancement exhibit local minima at the percolation threshold. Experimental observations, through theoretical corroboration, indicate a decrease of the SP localization in metal composites at critical concentrations with maxima in localization at concentrations away from the critical metal concentration. This effect runs opposite to the intuitive notion that stronger interactions lead to increase in localization. A possible explanation for this behavior is the existence of delocalized SP states, due to the local current conservation. Those states are present in the spectra for all metal concentrations and for metal-dielectric systems of any dimension. The possibility for this coexistence of both localized and delocalized SP modes has important ramifications in the understanding and applications of random metal films. Fine tuning the geometrical structure of the metal composites may allow for tuning of the localization properties of the excited SP modes, which may improve the enhancement of various linear and nonlinear optical processes.

\section{Acknowledgments}

Research partly supported by the U.S. Department of Energy, Basic Energy Sciences, Materials Sciences and Engineering Division, through Oak Ridge National Laboratory, U.S. Department of Energy (KS) and the Louisiana Board of Regents and NSF under Contracts nos. LEQSF(2011-14)-RD-A-18 and NSF(2010)-PFUND-20 (DAG).

\section{References}

[1] V. M. Shalaev, Nonlinear Optics of Random Media, Springer, Berlin, Germany, 2000.
[2] D. A. Genov, A. K. Sarychev, and V. M. Shalaev, "Plasmon localization and local field distribution in metal-dielectric films," Physical Review E, vol. 67, no. 5, Article ID 056611, 10 pages, 2003.

[3] D. Stauffer and A. Aharony, Introduction to Percolation Theory, Taylor \& Francis, London, UK, 2nd edition, 1992.

[4] V. M. Markel and T. F. George, Eds., Optics of Nanostructured Materials, John Wiley \& Sons, New York, NY, USA, 2001.

[5] D. P. Tsai, J. Kovacs, Z. Wang et al., "Photon scanning tunneling microscopy images of optical excitations of fractal metal colloid clusters," Physical Review Letters, vol. 72, no. 26, pp. 4149-4152, 1994.

[6] S. Grésillon, L. Aigouy, A. C. Boccara et al., "Experimental observation of localized optical excitations in random metaldielectric films," Physical Review Letters, vol. 82, no. 22, pp. 4520-4523, 1999.

[7] S. Ducourtieux, V. A. Podolskiy, S. Grésillon et al., "Near-field optical studies of semicontinuous metal films," Physical Review $B$, vol. 64, no. 16, Article ID 165403, pp. 1654031-16540314, 2001.

[8] S. I. Bozhevolnyi, I. I. Smolyaninov, and A. V. Zayats, "Nearfield microscopy of surface-plasmon polaritons: localization and internal interface imaging," Physical Review B, vol. 51, no. 24, pp. 17916-17924, 1995.

[9] S. I. Bozhevolnyi, V. S. Volkov, and K. Leosson, "Localization and waveguiding of surface plasmon polaritons in random nanostructures," Physical Review Letters, vol. 89, no. 18, Article ID 186801, 4 pages, 2002.

[10] A. K. Sarychev and V. M. Shalaev, "Electromagnetic field fluctuations and optical nonlinearities in metal-dielectric composites," Physics Report, vol. 335, no. 6, pp. 275-371, 2000.

[11] M. I. Stockman, "Local fields' localization and chaos and nonlinear-optical enhancement in clusters and composites," in Optics of Nanostructured Materials, V. A. Markel and T. F. George, Eds., pp. 313-354, John Wiley \& Sons, New York, NY, USA, 2000.

[12] J. A. Sánchez-Gil, J. V. García-Ramos, and E. R. Méndez, "Near-field electromagnetic wave scattering from random selfaffine fractal metal surfaces: spectral dependence of local field enhancements and their statistics in connection with surfaceenhanced Raman scattering," Physical Review B, vol. 62, no. 15, pp. 10515-10525, 2000.

[13] L. Zekri, R. Bouamrane, N. Zekri, and F. Brouers, "Localization and absorption of the local field in two-dimensional composite metal-dielectric films at the percolation threshold," Journal of Physics Condensed Matter, vol. 12, no. 3, pp. 283291, 2000.

[14] D. A. Genov, A. K. Sarychev, V. M. Shalaev, and A. Wei, "Resonant Field Enhancements from Metal Nanoparticle Arrays," Nano Letters, vol. 4, no. 1, pp. 153-158, 2004.

[15] S. I. Bozhevolnyi and V. Coello, "Statistics of local field intensity enhancements at nanostructured surfaces investigated with a near-field optical microscope," Physical Review B, vol. 64, no. 11, Article ID 115414, pp. 1154141-1154147, 2001.

[16] K. Seal, M. A. Nelson, Z. C. Ying, D. A. Genov, A. K. Sarychev, and V. M. Shalaev, "Growth, morphology, and optical and electrical properties of semicontinuous metallic films," Physical Review B, vol. 67, no. 3, Article ID 035318, 13 pages, 2003.

[17] A. K. Sarychev, V. A. Shubin, and V. M. Shalaev, "Anderson localization of surface plasmons and nonlinear optics of metal-dielectric composites," Physical Review B, vol. 60, no. 24, pp. 16389-16408, 1999.

[18] M. I. Stockman, S. V. Faleev, and D. J. Bergman, "Localization versus delocalization of surface plasmons in nanosystems: can 
one state have both characteristics?" Physical Review Letters, vol. 87, no. 16, Article ID 167401, 4 pages, 2001.

[19] D. A. Genov, V. M. Shalaev, and A. K. Sarychev, "Surface plasmon excitation and correlation-induced localizationdelocalization transition in semicontinuous metal films," Physical Review B, vol. 72, no. 11, Article ID 113102, 4 pages, 2005.

[20] K. Seal, A. K. Sarychev, H. Noh et al., "Near-field intensity correlations in semicontinuous metal-dielectric films," Physical Review Letters, vol. 94, no. 22, Article ID 226101, 4 pages, 2005.

[21] Y. Yagil, P. Gadenne, C. Julien, and G. Deutscher, "Optical properties of thin semicontinuous gold films over a wavelength range of 2.5 to $500 \mu \mathrm{m}$," Physical Review B, vol. 46, no. 4, pp. 2503-2511, 1992.

[22] K. Seal, D. A. Genov, A. K. Sarychev et al., "Coexistence of localized and delocalized surface plasmon modes in percolating metal films," Physical Review Letters, vol. 97, no. 20, Article ID 206103, 2006.

[23] M. Kunz, G. A. Niklasson, and C. G. Granqvist, "Optical and electrical properties of sputter-deposited Al films close to the percolation threshold," Journal of Applied Physics, vol. 64, no. 7, pp. 3740-3742, 1988.

[24] D. A. Genov, K. Seal, X. Zhang et al., "Collective electronic states in inhomogeneous media at critical and subcritical metal concentrations," Physical Review B, vol. 75, no. 20, Article ID 201403, 2007.

[25] M. Gadenne, P. Gadenne, and J. Lafait, "Infrared optical absorption and fractal structure of granular media at percolation," Optics Communications, vol. 71, no. 5, pp. 273-278, 1989.

[26] F. Brouers, J. P. Clerc, G. Giraud, J. M. Laugier, and Z. A. Randriamantany, "Dielectric and optical properties close to the percolation threshold. II," Physical Review B, vol. 47, no. 2, pp. 666-673, 1993.

[27] J. B. Pendry, "Negative refraction makes a perfect lens," Physical Review Letters, vol. 85, no. 18, pp. 3966-3969, 2000.

[28] R. A. Shelby, D. R. Smith, and S. Schultz, "Experimental verification of a negative index of refraction," Science, vol. 292, no. 5514, pp. 77-79, 2001.

[29] N. Fang, H. Lee, C. Sun, and X. Zhang, "Sub-diffractionlimited optical imaging with a silver superlens," Science, vol. 308, no. 5721, pp. 534-537, 2005.

[30] Y. Lu, G. L. Liu, and L. P. Lee, "High-density silver nanoparticle film with temperature-controllable interparticle spacing for a tunable surface enhanced Raman scattering substrate," Nano Letters, vol. 5, no. 1, pp. 5-9, 2005.

[31] X. Xu, K. Seal, X. Xu et al., "High tunability of the surfaceenhanced Raman scattering response with a metal-multiferroic composite," Nano Letters, vol. 11, no. 3, pp. 1265-1269, 2011.

[32] J. E. Rowe, C. V. Shank, D. A. Zwemer, and C. A. Murray, "Ultrahigh-vacuum studies of enhanced Raman scattering from pyridine on Ag surfaces," Physical Review Letters, vol. 44, no. 26, pp. 1770-1773, 1980.

[33] K. Kneipp, Y. Wang, H. Kneipp et al., "Single molecule detection using surface-enhanced Raman scattering (SERS)," Physical Review Letters, vol. 78, no. 9, pp. 1667-1670, 1997.

[34] T. V. Teperik, F. J. García De Abajo, A. G. Borisov et al., "Omnidirectional absorption in nanostructured metal surfaces," Nature Photonics, vol. 2, no. 5, pp. 299-301, 2008.

[35] C. Hu, Z. Zhao, X. Chen, and X. Luo, "Realizing near-perfect absorption at visible frequencies," Optics Express, vol. 17, no. 13, pp. 11039-11044, 2009.
[36] V. N. Pustovif and G. A. Niklasson, "Observability of resonance optical structure in fractal metallic clusters," Journal of Applied Physics, vol. 90, no. 3, pp. 1275-1279, 2001.

[37] P. W. Anderson, "Absence of diffusion in certain random lattices," Physical Review, vol. 109, no. 5, pp. 1492-1505, 1958. 

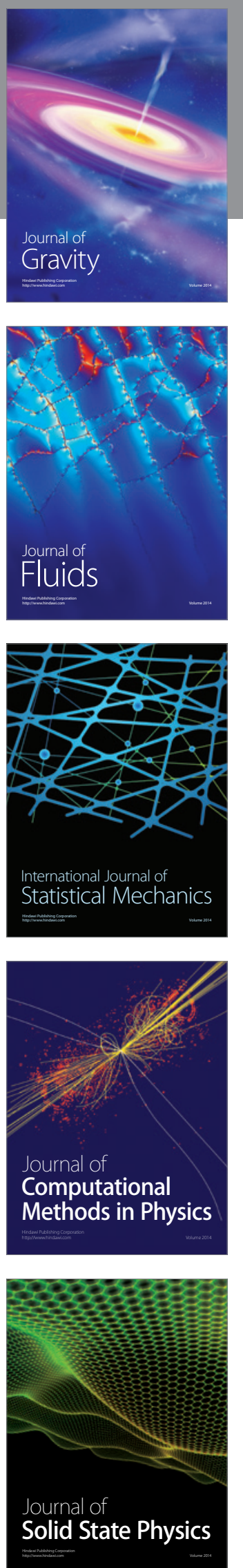

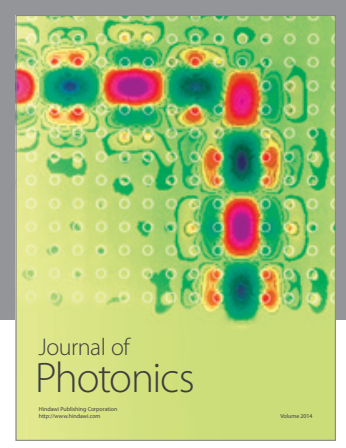

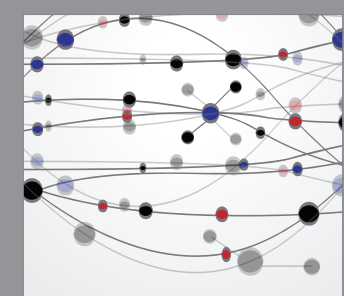

The Scientific World Journal
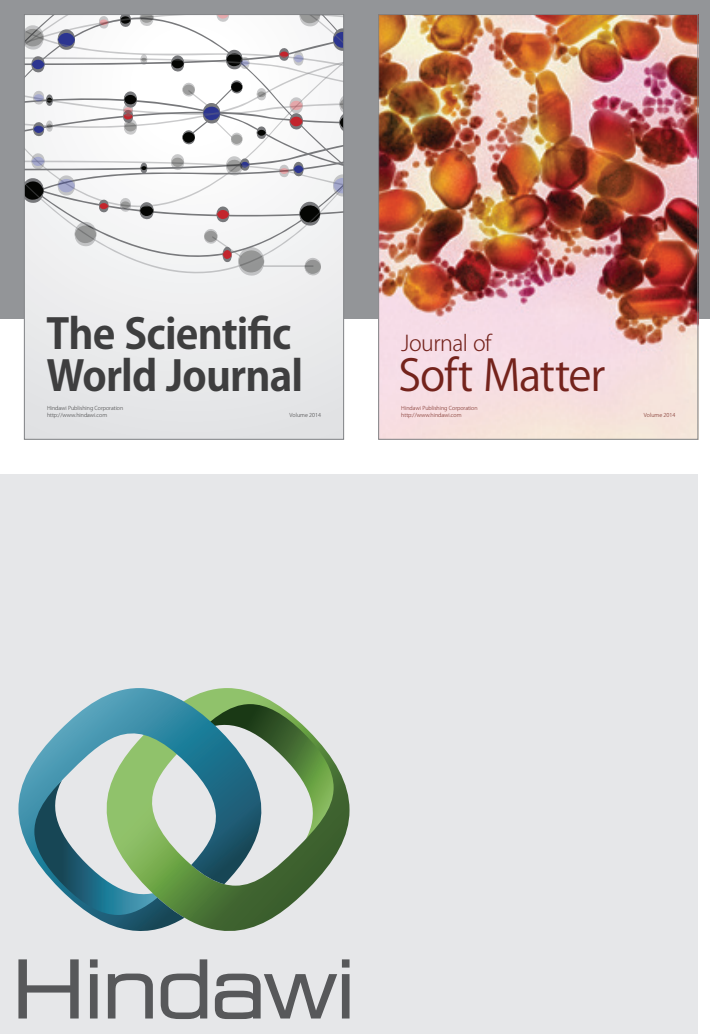

Submit your manuscripts at

http://www.hindawi.com
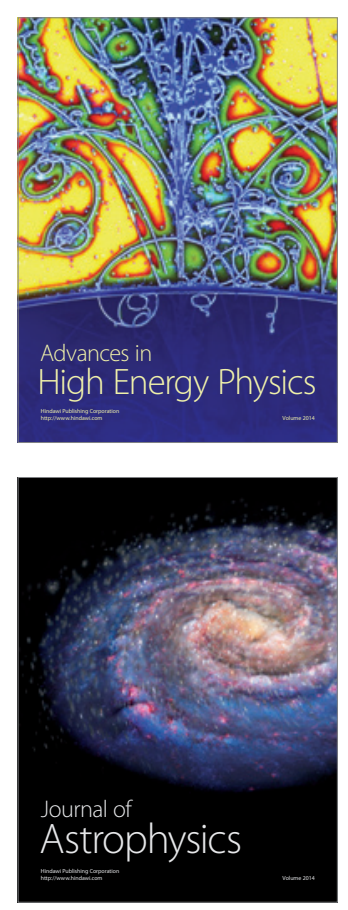
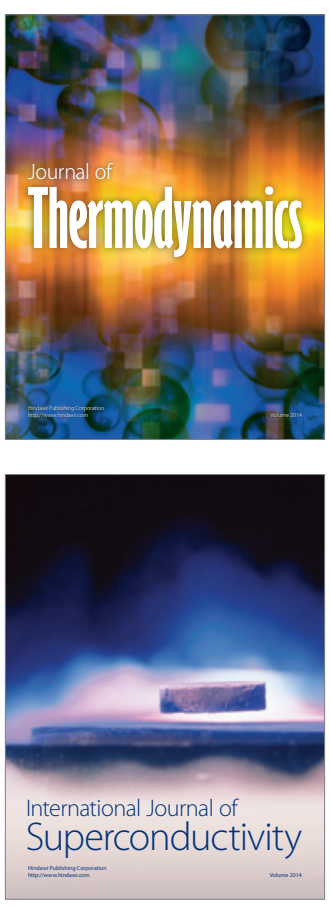
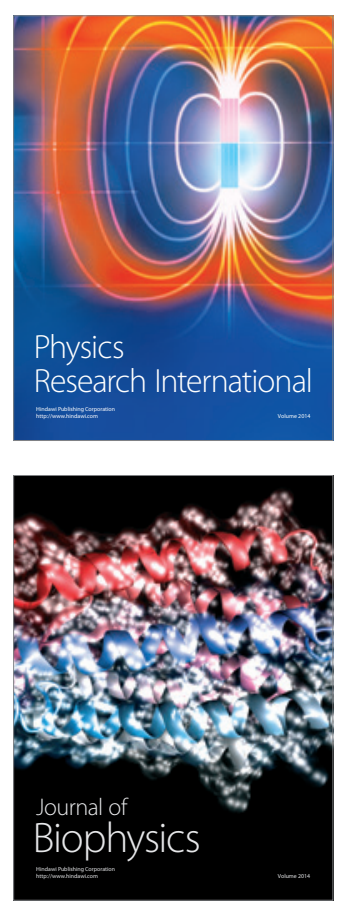
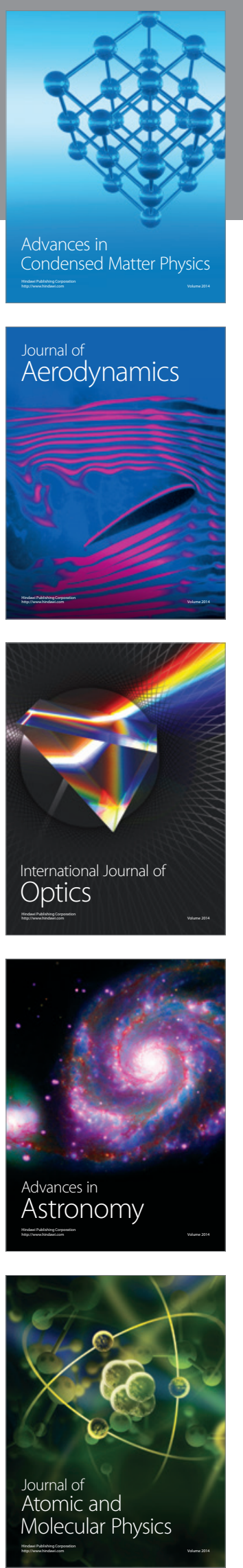